\title{
Die Autorinnen und Autoren
}

\author{
Dr. Séverine Adam \\ Université Paris-Sorbonne \\ Centre Malesherbes \\ 108, boulevard Malesherbes \\ F-75850 Paris Cedex 17 \\ adam.severine@googlemail.com
}

Hagen Augustin

Institut für Deutsche Sprache

R5, 6-13

D-68161 Mannheim

augustin@ids-mannheim.de

Prof. em. Dr. Péter Bassola

Universität Szeged

Germanistisches Institut

Egyetem utca 2

H-6722 Szeged

bassola@lit.u-szeged.hu

Dr. Edyta Błachut

Uniwersytet Wrocławski

Instytut Filologii Germańskiej

pl. Biskupa Nankiera $15 \mathrm{~b}$

PL-50-140 Wrocław

edyta.blachut@uni.wroc.pl

Prof. Dr. Lesław Cirko

Uniwersytet Wrocławski

Instytut Filologii Germańskiej

pl. Biskupa Nankiera $15 \mathrm{~b}$

PL-50-140 Wrocław

leslaw.cirko@uni.wroc.pl 
Viktória Dabóczi

Universität Siegen

Germanistisches Seminar

Adolf-Reichwein-Str. 2

D-57076 Siegen

daboczi@germanistik.uni-siegen.de

Prof. Dr. Martine Dalmas

Université Paris-Sorbonne

Centre Malesherbes

108, boulevard Malesherbes

F-75850 Paris Cedex 17

martine.dalmas@paris-sorbonne.fr

Dr. Cécile Delettres

8 , rue Lacépède

F-75005 Paris

cdelettres@yahoo.de

Prof. em. Dr. phil. Cathrine Fabricius-Hansen

Universitetet i Oslo

Institutt for litteraturvitenskap, områdestudier og europeiske språk (ILOS)

P.b. 1003 Blindern

N-0315 Oslo

c.f.hansen@ilos.uio.no

Dr. Beáta Gyuris

Ungarische Akademie der Wissenschaften

Institut für Linguistik

Benczúr utca 33

H-1068 Budapest

gyuris.beata@nytud.mta.hu

Dr. habil. Gottfried R. Marschall

vormals Université Paris-Sorbonne

Centre Malesherbes

108, boulevard Malesherbes

F-75850 Paris Cedex 17

gorumar@yahoo.de 
Dr. Horst Schwinn

Institut für Deutsche Sprache

R5, 6-13

D-68161 Mannheim

schwinn@ids-mannheim.de

Dr. Hélène Vinckel-Roisin

Université Paris-Sorbonne

Centre Malesherbes

108, boulevard Malesherbes

F-75850 Paris Cedex 17

helene.vinckel@paris-sorbonne.fr

Dr. Pál Uzonyi

Eötvös-Loránd-Universität

Germanistisches Institut

Rákóczi út 5

H-1088 Budapest

uzonyi.pal@btk.elte.hu 
